\title{
Variabilidad interanual de las anomalías de la temperatura superficial del mar en aguas cubanas y su relación con eventos El Niño-Oscilación del Sur (ENOS)
}

\author{
Benigno Hernández \\ Centro de Investigaciones Pesqueras, Ciudad de la Habana, Cuba \\ Dirección actual: CICESE, División de Oceanología, P.O. Box 434844, San Diego, \\ CA. 92143-4844 USA \\ E-mail: bhernan@cicese.mx
}

Recibido: 23 marzo 2001; versión corregida: 14 enero 2002; aceptado: 28 agosto 2002

\begin{abstract}
RESUMEN. Se analiza la serie de tiempo de anomalías de temperatura superficial del mar (TSM) en la cuadrícula cubana donde se muestran épocas frías y cálidas de las aguas alrededor de Cuba. Se comparan las anomalías de TSM en la región Niño 3 con las TSM de las aguas cubanas teniendo una alta correlación con un desfase de 4-5 meses. Se analizan las anomalías de TSM y la temperatura del aire en algunas de las estaciones meteorológicas costeras cubanas, así como la influencia de las anomalías de TSM sobre el desove y reclutamiento de la langosta espinosa Panulirus argus en la plataforma cubana, todas con alto nivel de significación estadística pero con meses de desfase.
\end{abstract}

Palabras claves: temperatura superficial del mar (TSM), ENOS, langosta, Cuba.

\section{Sea surface temperature anomalies interannual variability in Cuban waters and their relationship with El Niño-Southern Oscillation (ENSO) events}

\begin{abstract}
Sea surface temperature (SST) anomalies time series in the Cuban waters grid is analyzed. Cold and warm events in times series are shown. SST anomalies are compared with ENSO 3 region in the equatorial Pacific with SST anomalies Cuban waters having a high correlation with 4-5 months of lag. We are also analyzed the relationships between the SST anomalies and air surface temperature in some of the Cubans coastal meteorological data are analyzed, as well as the influence of the SST anomalies, with the spawns and recruitment of the spiny lobster Panulirus argus of the Cuban region, all with a good level of statistical significance with lags.
\end{abstract}

Key words: sea surface temperature (SST), ENSO, lobster, Cuba.

\section{INTRODUCCIÓN}

La temperatura superficial del mar (TSM) es uno de los más importantes predictores mensuales o estacionales del clima a nivel mundial (Tokioka, 1983). Diversos investigadores la utilizan en modelos estocásticos, ya sea para brindar pronósticos de la interacción océano-atmósfera, como para ser utilizada con enfoques multivariados en la biología pesquera de especies de interés comercial.

Uno de los eventos de la variabilidad climática a escala global donde se utiliza la TSM como uno de sus principales predictores es el fenómeno El
Niño-Oscilación del Sur (ENOS). Hoy en día, los trabajos de predicción de TSM en el Pacífico ecuatorial son cada vez más actuales, ya que son conocidas las pérdidas millonarias que ocurren por los efectos de este fenómeno en Perú, Chile, Ecuador, Brasil u otras regiones del mundo. Existe una serie de trabajos de predicción dentro de los cuales destacan los de Cane et al. (1986), Enfield (1995), Ji y Leetmaa (1995), Ji et al. (1995), Reynolds y Smith (1994), Smith et al. (1995) y, Zebiak y Cane (1995).

Regiones aledañas al océano Pacífico, como las 
cuencas del Indico y Atlántico han cobrado interés por su probada teleconexión con el Pacífico, específicamente la cuenca del Caribe (Roger, 1984; Enfield y Mayer, 1996), donde recién comienzan los estudios de predicción de TSM en el Atlántico a partir de los efectos del fenómeno ENOS en el Pacífico.

Además de lo anterior, se han elaborado trabajos donde se utiliza la TSM como predictora de lluvias y sequías en distintas regiones del mundo, como los de Ropelewski y Halpert (1987), Hastenrath et al. (1995) y Shabbar y Barnston (1996) en Sudáfrica; Hastenrath et al. (1984), Hastenrath (1990) y Marengo et al. (1993) en pronósticos de lluvias al NE de Brasil.

Existen otros pronósticos aún más complejos donde la TSM es la principal variable predictora, como ocurre con los modelos predictivos de huracanes en la cuenca del Caribe (Gray et al., 1993, 1994). Sin embargo, uno de los más importantes es el de Barnett y Preisendorfer (1987), donde pronostica la temperatura del aire para todas las regiones de Estados Unidos, utilizando la TSM y la presión atmosférica.

Por otra parte, son muchos los trabajos que utilizan la TSM como variable principal en el pronóstico del reclutamiento y captura de especies de interés comercial, como los relacionados con la pesquería de la anchoveta en Perú (Pauly y Tsukayama, 1983; Mendelssohn y Mendo 1987); de la sardina en la Corriente de California (Parrish et al., 1981, 1983; LLuch-Belda et al., 1989, 1991, 1992a, 1992b), donde se exponen y discuten varias estrategias en la relación entre factores hidroambientales y su comportamiento biológico-pesquero; y de la langosta en Australia (Pearce y Philips, 1988). Sin embargo, en Cuba por no contar con una serie de tiempo mensual de TSM extensa, tanto para aguas oceánicas como para plataforma, no existe un trabajo que los integre y solo se reducen a análisis estacionales y de oceanografía descriptiva (García, 1981a, 1981b; Siam y García, 1981).

Según Hela y Laevastu (1961), la TSM, independientemente que sea la que mejor refleja o determina la incidencia de los factores ambientales en el mar, es la que sirve como indicador de los cambios en las condiciones ecológicas. Por esta razón, los resultados del presente trabajo pueden servir para tener una mejor visión del hidroclima costero y del comportamiento de varias pesquerías atendiendo a su ciclo reproductivo anual, como la langosta y los túnidos, tanto en la plataforma como en aguas oceánicas y comparar a su vez, la tendencia que pudiera existir entre algunos datos meteorológicos costeros y la TSM. Por esta razón, se considera que la obtención de la TSM en la región de Cuba, sitúa a las investigaciones hidrometeorológicas de interés pesquero y de variabilidad climática, en una situación ventajosa entre otros países de la región del Gran Caribe.

\section{MATERIALES Y MÉTODOS}

Los datos mensuales de TSM y sus anomalías fueron aportados por el Dr. Richard Reynolds (Coupled Model Project, National Meteorological Center, NOAA), desde enero de 1970 a agosto de 1998, para el cuadrante Cuba ubicado entre los $17^{\circ}-25^{\circ} \mathrm{N}$ y $73^{\circ}$ $87^{\circ} \mathrm{W}$ en el Mar Caribe noroccidental (Fig. 1).

Se utilizaron tres técnicas específicas de análisis: los datos in situ, por satélites y por blended analysis o análisis combinado. Los datos in situ provinieron de observaciones de la superficie marina en una celda de $2^{\circ}$ de latitud por $2^{\circ}$ de longitud de los archivos del Centro Meteorológico Nacional de la NOAA, obtenidos por todos los barcos y boyas que entran al Sistema Global de Telecomunicaciones. Esta información es diaria, con 10 horas como mínimo de registro y aproximadamente 100.000 horas por mes en las observaciones para todo el mundo (Reynolds, 1988; Reynolds y Marsico, 1993).

En las observaciones satelitales se utilizó además la técnica de Temperatura Superficial del Mar Multicanal (MCSST) de McLain et al. (1985) y Walton (1988), que es uno de los métodos más confiables y utiliza el sensor AVHRR (Advanced Very High Resolution Radiometer) del satélite en órbita polar de la NOAA. El análisis combinado de ambas fuentes se realizó utilizando la técnica estadística sugerida por Oort y Rasmusson (1971) en coordenadas periféricas (Reynolds, 1988; Reynolds y Marsico, 1993).

Los datos de TSM en la región del Niño 3 fueron ofrecidos por la misma fuente y además, por el Dr. David Enfield (AOML, NOAA), lo que brinda confiabilidad en los resultados comparativos. La anomalía de temperatura superficial $\left({ }^{\circ} \mathrm{C}\right)$ se definió como el valor del dato, menos la media mensual de los años analizados $\left(\mathrm{t}_{\mathrm{i}}-\mathrm{T}\right)$; donde: $\mathrm{t}_{\mathrm{i}}=1,2,3, \ldots \mathrm{n}$. La serie cronológica de TSM en el cuadrante Cuba se procesó estadísticamente con promedios móviles 12 


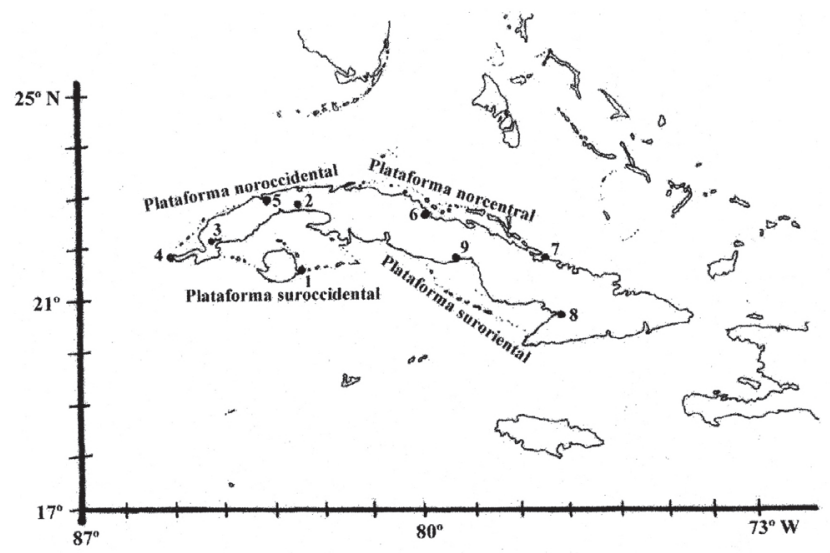

Figura 1. Ubicación del cuadrante Cuba y posición de las estaciones meteorológicas cercanas a la costa: 1 Punta del Este, 2 Batabanó, 3 Isabel Rubio, 4 Cabo de San Antonio, 5 Bahía Honda, 6 Caibarién, 7 Nuevitas, 8 Manzanillo y 9 Júcaro.

Figure 1. Cuban grill location and near shore meteorological station position: 1 Punta del Este, 2 Batabanó, 3 Isabel Rubio, 4 San Antonio Cape, 5 Bahía Honda, 6 Caibarien, 7 Nuevitas, 8 Manzanillo and 9 Júcaro.

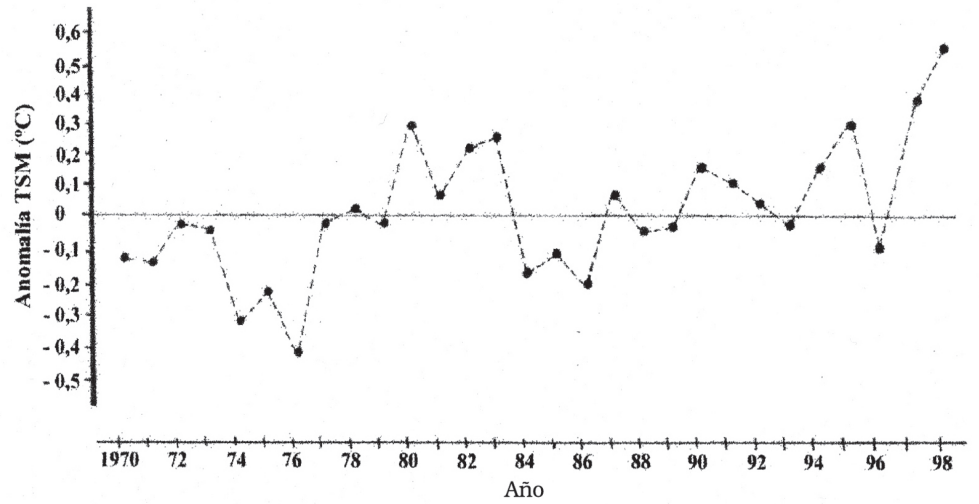

Figura 2. Promedio anual de las anomalías de temperatura superficial del mar $\left({ }^{\circ} \mathrm{C}\right)$ en el cuadrante Cuba. Figure 2. Sea surface temperature anomalies annual average in the Cuban grill.

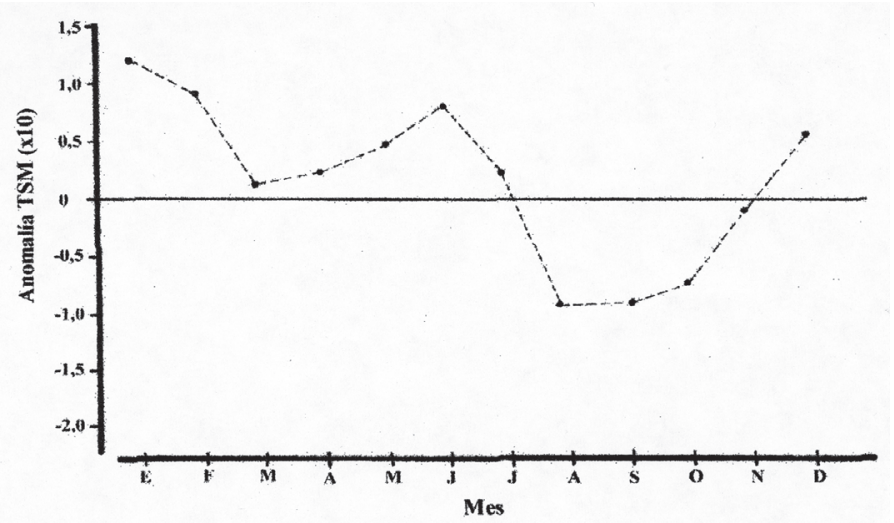

Figura 3. Estacionalidad de las anomalías de la TSM en el cuadrante Cuba en el período 1970-1998.

Figure 3. Cuban grill sea surface temperature seasonal anomalies in the 1970-1998 period. 
meses. También, se correlacionó la temperatura media mensual del aire en enero en las distintas estaciones meteorológicas costeras y la lluvia acumulada durante julio en la estación meteorológica de Batabanó con las anomalías de TSM del cuadrante Cuba.

Los registros de desembarques o capturas mensuales de langosta se obtuvieron a partir de la suma diaria de las tres asociaciones pesqueras que operan en el golfo de Batabanó (Isla de la Juventud, Pinar del Río y Batabanó), en la temporada de pesca (junio-febrero) desde 1969-70 a 1996-97 expresadas en miles de toneladas, por ser en esta región donde se realiza la pesquería más importante del país. Se relacionó igualmente, el comportamiento interanual de las anomalías de TSM con las capturas de langosta del golfo con dos años de desfase, para determinar el papel que pudieran ejercer las anomalías de TSM en el hidroclima, como efecto abiótico importante en los pronósticos del reclutamiento de esta especie (Puga et al., 1991).

\section{RESULTADOS}

El promedio anual de las anomalías de las TSM en la cuadrícula cubana desde 1970 a 1998 mostró que los años más fríos fueron entre 1974 y 1976, siendo este último el más frío de la serie analizada, con un promedio anual de $-0,42^{\circ} \mathrm{C}$ (Fig. 2). Por otra parte, los años de anomalías positivas o cálidas recayeron entre 1980 y 1983, 1995, 1997 y 1998, siendo este último el de mayor promedio, con $0,55^{\circ} \mathrm{C}$ de anomalía en agosto.

Las anomalías de la estacionalidad entre 1970 y 1998 indicaron que las mayores variaciones negativas o frías recayeron entre agosto y octubre, mientras que las anomalías positivas o cálidas se registraron en enero, febrero, junio y diciembre (Fig. 3).

La distribución de las anomalías negativas de la serie 1970-1998, mostró dos períodos significativos por debajo de la media. El primero de tres años y medio, abarcó desde el segundo semestre de 1973 hasta diciembre de 1976 y se caracterizó por presentar el mayor registro negativo de toda la serie en junio de $1976(-0,76)$. El segundo período significativo de anomalías negativas, abarcó desde el segundo semestre de 1984 hasta octubre de 1986, siendo este período de menor duración que el anterior (Fig. 4). Las anomalías positivas mostraron también dos períodos notables por encima de la media. El primero desde 1980 hasta el primer semestre de
1984, y el segundo entre 1990 y 1998. Durante casi toda la década de 1990 las anomalías de TSM fueron altas para la zona de estudio. La anomalía más alta de toda la serie ocurrió en junio de 1998 con 0,98 . En la década de los 70 las anomalías de TSM fueron más frías que las de los 80 y 90 .

La Figura 5a indicó el comportamiento de las anomalías de TSM en enero y la temperatura del aire en la estación meteorológica de Punta del Este, región suroccidental, desde 1975 hasta 1994. El coeficiente de correlación $r=0,62$ fue significativo ( $P$ $<0,05)$. Las Figuras $5 \mathrm{~b}$ a $5 \mathrm{e}$, muestran el comportamiento de las anomalías de TSM en enero (mes de mayor contraste térmico), con las temperaturas del aire superficial en las distintas estaciones meteorológicas. En las más cercanas al mar (Nuevitas y Punta del Este) el coeficiente de correlación fue más alto, mientras que en las más alejadas (Isabel Rubio y Batabanó), el coeficiente fue menor. Vale destacar la pronunciada anomalía negativa observada en todas las estaciones en 1976, 1981 y 1985, aún así se mantuvo una relación estrecha con tendencia al ascenso desde 1985 entre ambas temperaturas.

La Figura 6 mostró la relación entre las anomalías de TSM y la lluvia en julio, desde 1970 hasta 1993-94 reportada en la estación meteorológica de Batabanó en la región suroccidental de la isla. Desde el punto de vista climático ambas variables presentaron las mismas tendencias. El comportamiento frío de la TSM en los períodos 1974-1976 y 19841986 coincidió con anomalías negativas de lluvia en esos mismos años, mientras que en los años cálidos (1978-1983 y 1987-1989), las anomalías de lluvia fueron positivas. El coeficiente de determinación entre la temperatura y lluvia en la estación meteorológica de Batabanó fue de 0,59 lo que comprueba la importancia de esta variable en las relaciones hidroclimáticas.

La Figura 7 muestra la correlación cruzada entre las anomalías de TSM en la región Niño 3 y las anomalías de TSM en la cuadrícula cubana durante los últimos seis eventos ENOS. Esta muestra una correlación negativa significativa $(-0,64)$ con dos meses de desfase y una correlación positiva significativa $(0,71)$ cuatro meses de desfase después; es decir, seis meses a partir del inicio de un sobrecalentamiento en la región Niño 3 provocado por el inicio de un evento ENOS, concordando con lo señalado para la cuenca del Caribe (Enfield y Mayer, 1997).

La Figura 8 muestra el gráfico de dispersión en- 

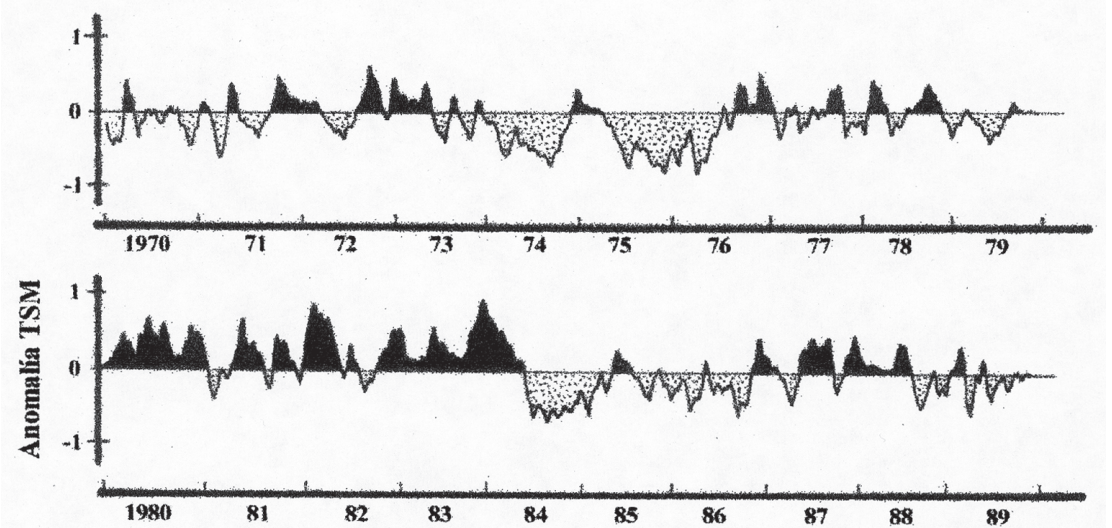

Figura 4. Serie de tiempo de las anomalías mensuales de TSM desde 1970 hasta 1998 en el

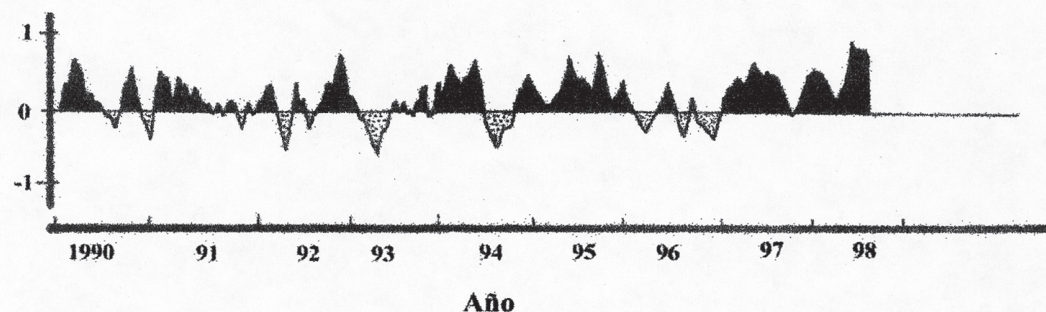
cuadrante Cuba.

Figure 4. Sea surface temperature anomalies time series for 1970 . 1998 in the Cuban grill.
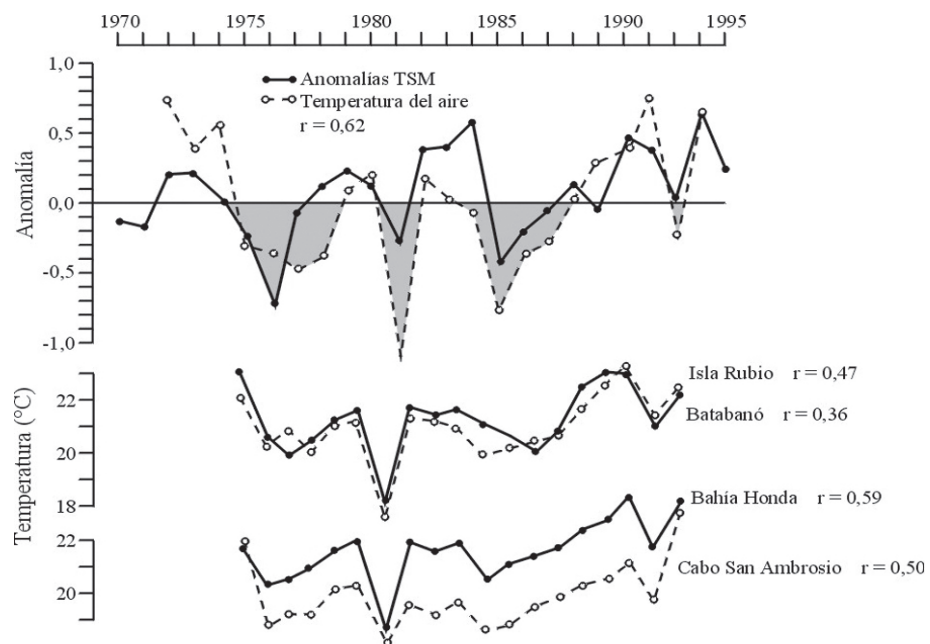

Figura 5. Comportamiento interanual de los eneros entre las anomalías de TSM y la temperatura superficial del aire (TSA) en las estaciones meteorológicas cercanas a la plataforma marina cubana desde 1970 hasta 1994. a) TSM vs. TSA en Punta del Este, b) TSM vs. TSA en I. Rubio y TSM vs. TSA en Batabanó, c) TSM vs. TSA en B. Honda y TSM vs. TSA en Cabo de San Antonio, d) TSM vs. TSA en Caibarién y TSM vs. TSA en Nuevitas, y e) TSM vs. TSA en Manzanillo y TSM vs. TSA en Júcaro.

Figure 5. January interannual behavior between sea surface temperature anomalies (SST) and air surface temperature (AST) in near shore meteorological station for 1970-1998. a) SST vs. AST in Punta del Este, b) SST vs. AST in I. Rubio and SST vs. AST in Batabano, c) SST vs. AST in B. Honda and SST vs. AST in San Antonio Cape, d) SST vs. AST in Caibarien and SST vs. AST in Nuevitas, and e) SST vs. AST in Manzanillo and SST vs. AST in Jucaro. 


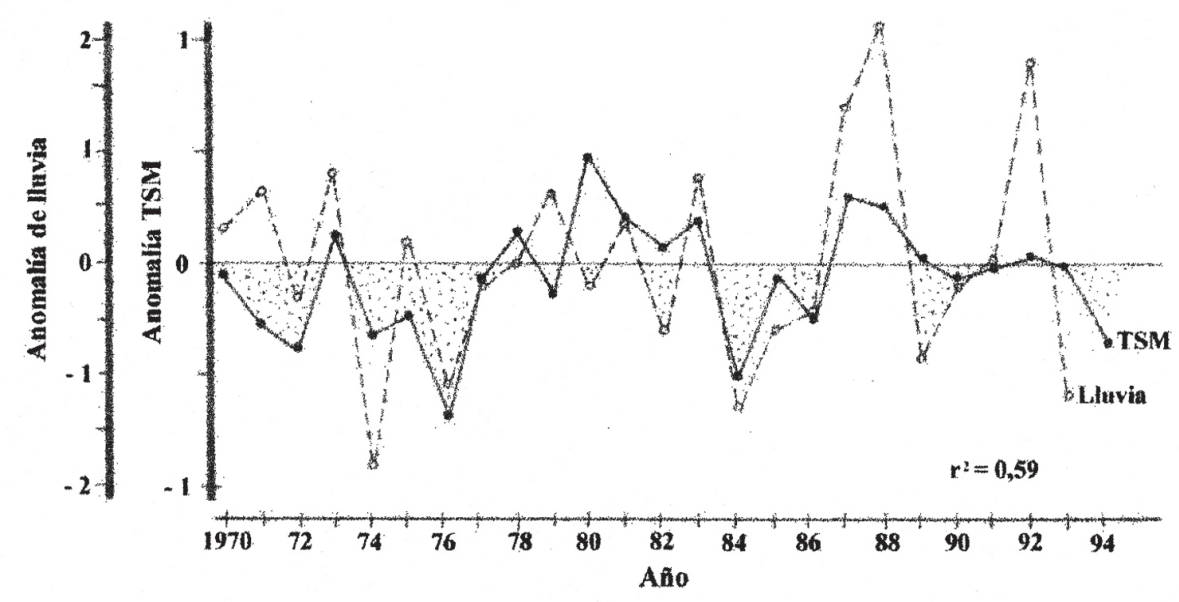

Figura 6. Comportamiento de las anomalías de TSM (cuadrante Cuba) y de lluvia (mm), registradas en julio en la estación meteorológica de Batabanó. El coeficiente de determinación entre ambas variables fue de 0,59 explicando un alto porcentaje de la lluvia registrada.

Figure 6. Sea surface temperature behavior (Cuban grill) and rain ( $\mathrm{mm}$ ) registered in July in Batabano meteorological station. Determination coefficient $\left(r^{2}\right)$ between both variables was 0.59 explaining a high percentage of the registered rain.

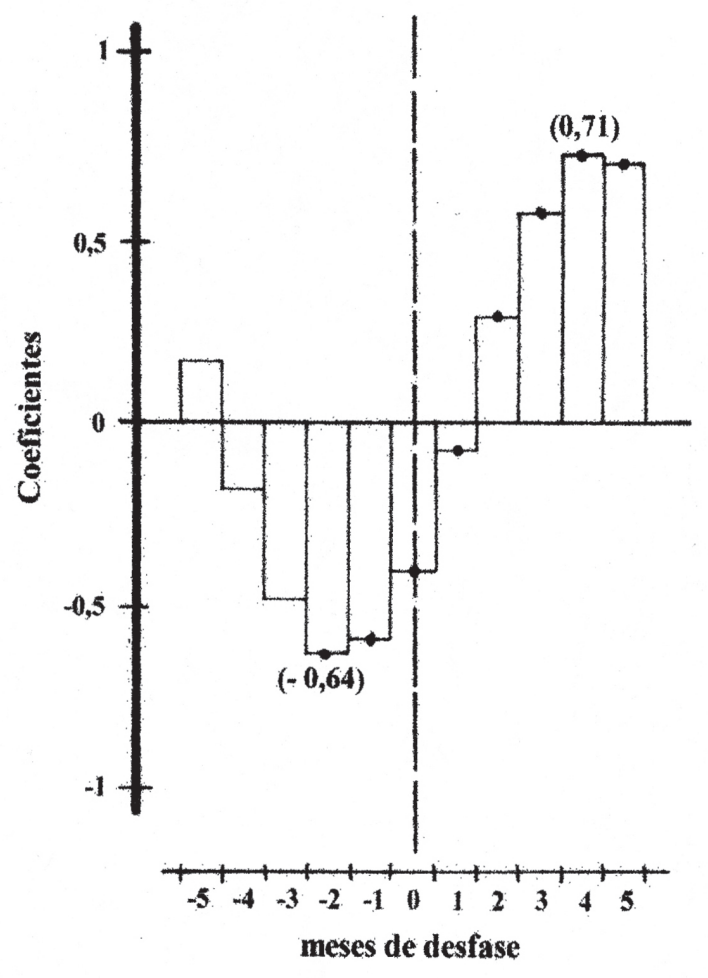

Figura 7. Correlación cruzada entre las anomalías de TSM en la región Niño 3 y las del cuadrante Cuba durante el período 1970-1994.

Figure 7. Cross correlation between SST anomalies in Niño 3 and the Cuban grill for 1970-1994. 


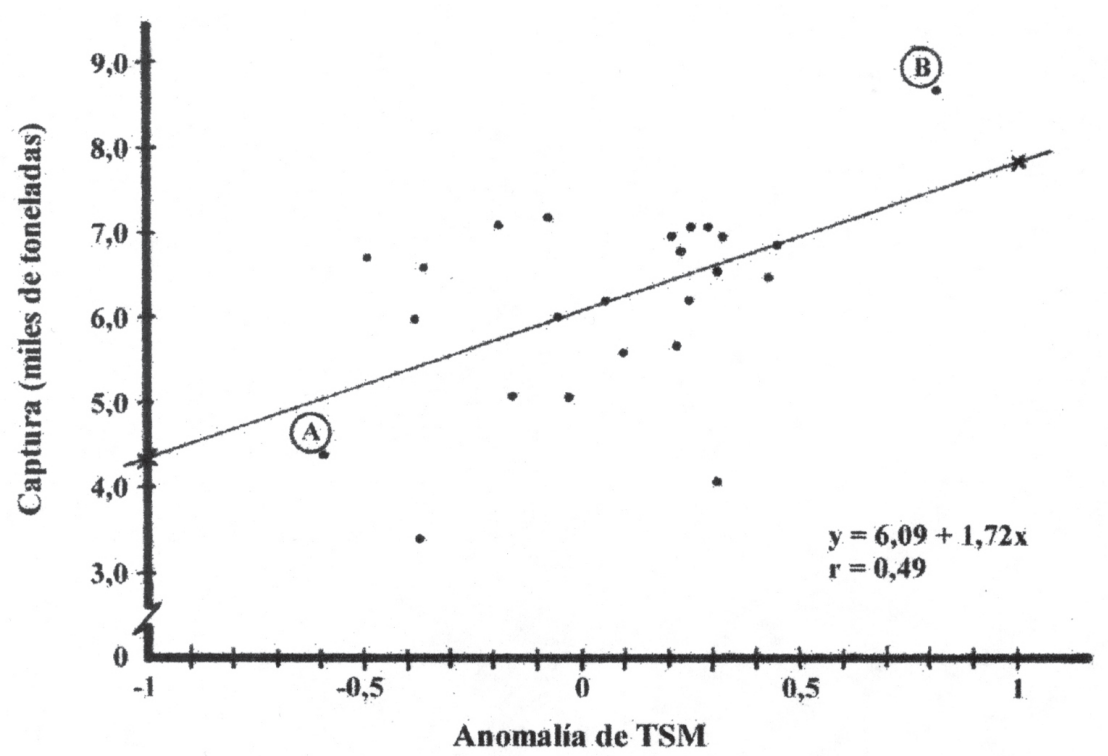

Figura 8. Gráfico de dispersión con dos años de desfase entre las anomalías de TSM en el cuadrante Cuba y las capturas de langosta Panulirus argus en el golfo de Batabanó. A. Representa el punto extremo de anomalías negativas de TSM en 1975-76 y las capturas de langosta en 1977-78, B. Representa el punto extremo de anomalías positivas de TSM en 1983-84 y las capturas de langosta en 1985-86.

Figure 8. Two year lack dispersion graph between sea surface temperature anomalies in Cuban grill and spiny lobster landings in the gulf of Batabano. A. Represent SST negative anomalies extreme point in 1975-76 and lobster landing in 1977-78, B. Represent SST positive anomalies extreme point in 1983-84 and lobster landing in 1985-86.

tre las anomalías de TSM en diciembre con dos años de antelación con respecto a las capturas totales de langosta espinosa Panulirus argus en el golfo de Batabanó. Las anomalías negativas de la TSM en diciembre de 1975 y la captura de langosta en la temporada de pesca 1977-78, se representan en el punto A con una tendencia a disminuir dos años después de un mínimo en la TSM. El punto B está representado por las anomalías positivas de TSM en 1983 y las capturas en el golfo en 1985-86. El resto de los puntos corresponden a las TSM y las capturas dos años después, en el período 1972-73 a 199697. Como efecto abiótico, este parámetro puede ser de gran utilidad si se incluye entre las variables ambientales para hacer futuros pronósticos de reclutamiento de la langosta señalado anteriormente por Puga et al. (1991). La Tabla 1 señala los coeficientes de correlación entre las temperaturas mensuales y las capturas en el golfo de Batabanó, mostrando un ascenso a partir de septiembre y llegando a ser significativo en diciembre con $(r=0,49$ $\mathrm{P}<0,05)$.

\section{DISCUSIÓN}

La serie de TSM en aguas cubanas indica un comportamiento anómalo durante los últimos seis eventos ENOS. Esta teleconexión con las aguas del Pacífico ecuatorial es debida principalmente a que la región de estudio está "conectada" bajo el régimen de los alisios del NE y es forzada a reducir la velocidad del viento superficial asociada con los cambios en la troposfera alta inducidos por los eventos ENOS que ocurren en el Pacífico ecuatorial. Los cambios en la velocidad del viento afectan la temperatura en la capa de mezcla a través de la evaporación y la transferencia de calor sensible decreciendo en orden de importancia (Enfield y Mayer, 1996). Los resultados sugieren que la variabilidad de TSM en el Pacífico está fuertemente relacionada con las TSM de la región cubana; por lo tanto, monitoreando la TSM en la región Niño 3 podría de alguna manera predecir el posible comportamiento de las aguas cubanas con 5-6 meses de desfase. 
Tabla 1. Coeficientes de correlación mensual entre la TSM y las capturas de langosta en el Golfo de Batabanó. Table 1. Monthly coefficients correlation between TSM and Gulf of Batabano lobster captures.

\begin{tabular}{|ccccccccccccc|}
\hline & $\mathrm{E}$ & $\mathrm{F}$ & $\mathrm{M}$ & $\mathrm{A}$ & $\mathrm{M}$ & $\mathrm{J}$ & $\mathrm{J}$ & $\mathrm{A}$ & $\mathrm{S}$ & $\mathrm{O}$ & $\mathrm{N}$ & $\mathrm{D}$ \\
\hline $\mathrm{r}$ & 0,04 & $-0,08$ & $-0,1$ & 0,06 & 0,07 & 0,03 & 0 & 0,02 & 0,11 & 0,22 & 0,32 & 0,49 \\
\hline
\end{tabular}

Durante el evento ENOS de 1972-73, se registró la mayor anomalía positiva a fines de 1972 que duró hasta el segundo semestre de 1973. En el evento 1976-77 se reportaron las máximas anomalías negativas de la serie y el calentamiento máximo se produjo a fines de 1976 y primeros meses de 1977. Las anomalías negativas del 1975-76 coincidieron casi un año después del período frío de 1975 en la región Niño 3 del Pacífico ecuatorial.

Caso aparte merece el evento ENOS de 198283. Previo a un intenso calentamiento que abarcó el primer semestre de 1982, la TSM alcanzó valores positivos significativos a partir de diciembre de 1982 y durante todo 1983, registrándose en diciembre de 1983 y en enero de 1984, uno de los valores más altos de toda la serie, descendiendo bruscamente durante los primeros seis meses de 1984. Las condiciones hidroclimáticas fueron severas durante este último episodio, con reportes de penetraciones marinas en el malecón del litoral habanero (E. Rodríguez, Weather Channel, com. pers.), escasa presencia de algunas especies marinas en aguas cubanas y registro de algunas especies de celenterados, como medusas macroplanctónicas de otras regiones, en la plataforma marina cubana (Raúl González, CIP, com. pers.).

El episodio ENOS 1987-88 tuvo menos repercusión con relación a las TSM de las aguas cubanas. A partir de un calentamiento previo a fines de 1986 y principios de 1987, los valores de anomalías positivas se extendieron durante el resto de $1987 \mathrm{y}$ hasta agosto de 1988. Las anomalías positivas no llegaron a ser significativas, comparadas con las del evento ENOS 1982-84 por lo que no tuvo un cambio significativo en la hidrometeorología de las costas cubanas.

El evento ENOS 1992-93 presentó algunas anomalías positivas máximas hacia fines de 1992, enero de 1993 y primeros seis meses de 1994. En 1993 se manifestó otro gran descenso brusco en las anomalías de TSM que abarcó de marzo a julio, pero no fue tan pronunciado como el de 1984. El descenso de las anomalías de TSM a condiciones normales en la región Niño 3 registradas en la segunda mitad de 1992 (Climate Diagnostic Bulletin, 1993), al parecer, se reflejó en un enfriamiento de las aguas en la región de Cuba en el segundo trimestre de 1993.

Durante el último evento ENOS 1997-98, las aguas se mantuvieron con anomalías positivas desde el inicio de 1997 y durante 1998, reportándose en junio la anomalía más alta de TSM. Como consecuencia del evento ENOS, el Instituto de Meteorología de Cuba informó de situaciones anómalas en la temporada invernal 1997-98 con un aumento en la entrada de frentes fríos, lluvias que superaron los valores normales en la región occidental cubana y fuertes penetraciones marinas en el malecón del litoral habanero. Es importante destacar que las anomalías de TSM fueron completamente distintas en cada uno de los eventos ENOS.

Las anomalías de TSM son una de las condicionantes físicas que están intrínsecamente ligadas al mar, al afectar directa y/o indirectamente a través de varias vías los cambios en los factores hidroambientales, como el régimen de corrientes geostróficas, profundidad de inicio de la termoclina, estructura homotermal y transporte (Hela y Laevastu, 1961). Las fluctuaciones en las anomalías de TSM ejercen igualmente, influencia directa sobre la comunidad bentónica y de corales (Glynn, 1990; Arntz et al., 1991); retención y reclutamiento larval (Pringle, 1986); desove, reclutamiento y pesquerías de cualquier especie (Pearce y Philips, 1988), adelantando o retrasando su ciclo estacional. Dow (1980) encontró una asociación estrecha entre la TSM y las variaciones en las capturas de la langosta Homarus americanus en la plataforma de Maine (Estados Unidos). Desde el punto de vista biológico, puede tener diversos efectos en peces, crustáceos y demás componentes de la fauna a través de los mecanismos receptores del sistema nervioso central, modificando los estímulos, los procesos metabólicos y finalizando muchas veces, con una actividad migratoria que los mueve hacia zonas de "máximo confort" (Cayré, 1990).

El análisis de los parámetros biológico-pesqueros calculados en función de la actividad pesquera de la langosta espinosa Panulirus argus en aguas cuba- 
nas, indica que su reclutamiento radica fundamentalmente en el número de ejemplares de 2 años de edad, mientras que las condiciones ambientales imperantes en períodos previos al reclutamiento a la pesquería, en combinación con la masa desovadora, influyen significativamente en la magnitud del reclutamiento resultante y su efecto subsiguiente sobre las capturas de este crustáceo (Puga et al., 1991).

Según (Evans y Evans, 1995) los cambios interanuales en la abundancia de Panulirus argus y $P$. guttatus, sugieren una asociación con el promedio de TSM en la plataforma de las Bermudas. Evans et al. (1995) formularon una hipótesis donde explican que las anomalías de TSM son importantes en la determinación del tiempo y nivel de reclutamiento anual en las pesquerías en las Bermudas. El aumento en el coeficiente de correlación (Tabla 1) no es extraño si se tiene en cuenta que a partir de septiembre la curva del ciclo estacional de TSM comienza a ascender hasta ser positiva justamente en diciembre. José Gómez (CIP, com. pers.) propone la temperatura del mar de diciembre en combinación con las hembras ovígeras, para detectar un adelanto o retraso en el desove de la langosta (marzomayo) en el golfo de Batabanó.

En este sentido, los resultados expuestos deben tomarse con cautela ya que los datos de la TSM corresponden a promedios mensuales en cuadrículas de $2^{\circ} \times 2^{\circ}$ grados, lo cual requiere de una serie de tiempo más larga unido a estudios multidisciplinarios para probar modelos predictivos que revelen relaciones entre las variaciones climáticas a largo plazo o de baja frecuencia y variaciones biológicas de alta frecuencia. Ambos resultados servirán para delinear la definición de cambio.

\section{CONCLUSIONES}

En la serie de tiempo mensual de TSM alrededor de la isla se determinaron dos épocas de anomalías negativas importantes: la primera entre 1973 y 1976 , y la segunda entre 1984 y 1986. Las etapas de las anomalías positivas de TSM abarcaron desde 1977 hasta 1983, desde 1989 hasta fines de 1992 y por último, las más cálidas desde 1997 hasta mediados de 1998. El mes más frío fue junio de $1976(-0,76)$ y el más cálido, junio de 1998 (0,98).

Las anomalías negativas de TSM generalmente respondieron a temperaturas frías del aire y poca lluvia, mientras que los períodos con anomalías po- sitivas correspondieron a temperaturas elevadas con mayores probabilidades de lluvia.

En los últimos seis eventos ENOS se comprobó que las altas temperaturas del mar en el Pacífico ecuatorial se reflejaron también en aguas de Cuba, pero con menor intensidad y se correlacionaron significativamente con la región de Niño 3, con 5-6 meses de desfase.

Las anomalías de TSM de diciembre se correlacionaron con las capturas de langosta en el golfo de Batabanó con dos años de desfase.

\section{REFERENCIAS}

Arntz, W.E., J. Tarazona, V.A. Gallardo, L.A. Flores y H. Salzwedel. 1991. Benthos communities in oxygen deficient shelf and upper slope areas of the Peruvian and Chilean Pacific coast, and changes caused by El Niño. En: R.V. Tyson and T.H. Pearson (eds.). Modern and ancient continental shelf anoxia. Geol. Soc. Spec. Publ., 58: 131-154.

Barnett, T. y R. Preisendorfer. 1987. Origins and levels of monthly and seasonal forecast skill for US surface air temperature determine by Canonical Correlation Analysis. Mon. Wea. Rew., 115(9): 1825-1850.

Cane, M., S. Zebiak y C. Dolan. 1986. Experimental forecast of El Niño. Nature, 321: 827-832.

Cayré, R. 1990. Les migrations: un comportement déclenché et guide par l'environnant. ICCAT, 32(1): 158-168.

Climatic Diagnostic Bulletin. 1993. Climate Analysis Center. US. Dep. Comm. NOAA, 93: 1-12.

Dow, R.L. 1980. The clawed lobster fisheries. En: J.S. Cobb and B.F. Phillips (eds.). The biology and management of lobsters. Vol. II. Ecology and management. Academic Press, New York, pp. 265316.

Enfield, D.B. 1996. Relationships of inter-American rainfall to tropical Atlantic and Pacific SST variability. Geophys. Res. Lett., 23: 3505-3508.

Enfield, D.B. y M.A. Mayer. 1997. Tropical Atlantic SST variability and its relation to "El Niño/ Southern Oscillation”. J. Geophys. Res., 102: 929945. 
Evans, C.R. y A.J. Evans. 1995. Fishery ecology and spiny lobsters Panulirus argus and Panulirus guttatus (Latreille) at Bermuda: Estimates of sustainable yields and observations on trends in abundance. Fisheries Res., 24: 113-128.

Evans, C.R., A.P.M. Lockwood y A.J. Evans. 1995. Associations between sea temperature, catch per unit of fishing effort and yield in the Bermuda spiny lobster fishery 1975-1989. Freshwater Res., 46: 809-818.

García, C. 1981a. Temperatura de las aguas oceánicas de Cuba: I. Aguas superficiales. Rev. Cub. Inv. Pesq., 6(2): 1-15.

García, C. 1981b. Temperatura de las aguas oceánicas de Cuba: II. Aguas subsuperficiales. Rev. Cub. Inv. Pesq., 6(2): 16-35.

Glynn, P.W. 1990. Global ecological consequences of the 1982-83 El Niño-Southern Oscillation. Elsevier Oceanography Series (52): 584 pp.

Gray, W.M., C.W. Landsea, P.W. Mielke y K. Berri. 1993. Predicting Atlantic seasonal hurricane activity 6-11 month in advance. Weather Forecast, 7: 440-455.

Hastenrath, S. 1990. Prediction of northeast Brazil rainfall anomalies. J. Climate, 3(8): 893-904.

Hastenrath, S., L. Greischar y J. van Heerden. 1995. Prediction of the summer rainfall over South Africa. J. Climate, 8: 1511-1518.

Hastenrath, S., M.C. Wu y P.S. Chu. 1984. Towards the monitoring and prediction of north-east Brasil droughts. Quart. J. Roy. Meteorol. Soc., 110: 411425 .

Hela, I. y T. Laevastu. 1961. Fishery hydrography. Fishing News (Books). Ltd., London, 137 pp.

Houghton, R.W. 1991. The relationship of sea surface temperature to thermocline depth at annual and interannual time scale in the tropical Atlantic Ocean. J. Geophys. Res., 96: 15173-15185.

Ji, M., A. Leetmaa y J. Derber. 1995. An ocean analysis for seasonal to interannual climate studies. Mon. Wea. Rew., 123: 460-481.

Lluck, D., R.J.M. Crawford, T. Kawasaki, R. Mc Call, R. Parrish, R.A. Schwartlose y P.E. Smith. 1989. Word-wide fluctuation of sardine and anchovy stock: the regime problem. S. Afr. J. Marine Sci., 8: 195-205.
Lluck, D., D.B. Lluck-Cota, S. Hernández-Vázquez, C.A. Salinas y R.A. Schwartlose. 1991. Sardine and anchovy spawning as related to temperature and upwelling in the California Current System. CalCOFI Rep., 32: 105-111.

Lluck, D., D.B. Lluck-Cota, S. Hernández-Vázquez y C.A. Salinas. 1992a. Sardine population expansion in eastern boundary systems of the Pacific Ocean as related to sea surface temperature. En: A.I.L. Payne, K.H. Brink, K.H. Man and R. Hilborn (eds.). Benguela Trophic Functioning. S. Afr. J. Marine Sci., 12: 144-147.

Lluck, D., R.A. Schwartlose, R. Serra, R. Parrish, T. Kawasaki, D. Hedgecock y R.J.M. Crawford. 1992b. Sardine and anchovy regime fluctuations of abundance in four regions of the world oceans: a workshop report. Fisheries Oceanogr., 1(4): 339-347.

Marengo, J.A., L.M. Druyan y S. Hastenrath. 1993. Observational and modeling studies of Amazonian interannual climate variability. Climate Change, 23: 267-286.

McLain, E.P., W.G. Pichel y C.C. Walton. 1985. Comparative performance of AVHRR based multichannel sea surface temperatures over the ocean. Mon. Wea. Rew., 112: 303-313.

Mendelssohn, R. y J. Mendo. 1987. Exploratory analysis of anchoveta recruitment off Peru and related environmental series. En: D. Pauly and I. Tsukayama (eds.). The Peruvian anchovy and its upwelling ecosystem: three decades of changes. Instituto del Mar de Peru (IMARPE), Callao, Peru; Deutsche Gesellschaft fur Technische Zusammenarbeit (GTZ), GmbH, Eschborn, Federal Republic of Germany; and International Center for Living Aquatic Resources Management, Manila, Philippines. ICLARM Studies and Review, 15, $351 \mathrm{pp}$.

Oort, A.H. y E.M. Rasmusson. 1971. Atmospheric circulation statistics. NOAA Professional Paper No. 5, 323 pp. (Available from US Government Printing Office, Washington. DC, 20402, Stock No. 03170045.)

Parrish, R.A., C.S. Nelson y A. Bakun. 1981. Transport mechanism and reproductive success of fishes in the California Current. Biol. Oceanogr., 1(2): 175-203.

Pauly, D. y I. Tsukayama. 1983. On the seasonal growth, monthly recruitment and monthly biomass 
of Peruvian anchovy (E. ringens) from 1961 to 1979. FAO Fish. Rep., 291(3): 987-1004.

Pearce, A. y B. Philips. 1988. ENSO events, the Leewin current, and larval recruitment of the western rock lobster. J. Cons. Int. Expl. Mer., 44: 18-26.

Pringle, J.D. 1986. California spiny lobster (Panulirus interruptus) larval retention and recruitment: a review and synthesis. Can. J. Fisheries Aquat. Sci., 43(11): 2142-2152.

Puga, R., M.E. de León y R. Cruz. 1991. Evaluación de la pesquería de langosta espinosa (Panulirus argus) en Cuba. Rev. Cub. Inv. Mar., 12: 286-292.

Reynolds, R.W. 1988. A real-time sea surface temperature analysis. J. Climate, 1: 75-86.

Reynolds, R.W. y D.C. Marsico. 1993. An improved real-time sea surface temperature analysis. J. Climate, 6(1): 114-119.

Reynolds, R.W. y T.M. Smith. 1994. Improved global sea surface temperature analysis using optimum interpolation. J. Climate, 7: 929-948.

Roger, J.C. 1984. The association between the North Atlantic Oscillation and the Southern Oscillation in the North Hemisphere. Mon. Wea. Rew., 112: 1999-2015.
Ropelewski, C.F. y M.S. Halpert. 1987. Global and regional scale precipitation patterns associated with the "El Niño/Southern Oscillation". Mon. Wea. Rew., 115: 1606-1626.

Siam, C. y C. García. 1981. Temperatura de las aguas oceánicas de Cuba: profundidad de inicio de la termoclina en las aguas oceánicas de Cuba. Rev. Cub. Inv. Pesq., 6(2): 36-49.

Tokioka, T. 1983. Influence of the ocean on the atmospheric global circulations and short-range climatic fluctuations. En: G.D. Sharp and J. Csirke (eds.). Proceeding of the expert consultation to examine change in abundance and species of neritic fish resources. San José de Costa Rica. FAO Fish. Rep., 291(3): 557-585.

Walton, C.C. 1988. Nonlinear multichannel algorithms for estimating sea surface temperature with AVHRR satellite data. J. Appl. Meteorol., 27: 115-124.

Williams, R.G. 1989. The influence of air-sea interaction on the ventilated thermocline. Amer. Meteorol. Soc., 19: 1255-1267.

Zebiak, S.E. y M.A. Cane. 1987. A model El NiñoSouthern Oscillation. Mon. Wea. Rev., 115: 22622278 . 